Review Article

\title{
Diagnostic and Therapeutic Potential of Exosomal MicroRNAs for Neurodegenerative Diseases
}

\author{
Miao He $\mathbb{D}^{1}{ }^{1}$ Hai-nan Zhang, ${ }^{1}$ Zhen-chu Tang, ${ }^{1}$ and Shu-guang Gao $\mathbb{D}^{2,3}$ \\ ${ }^{1}$ Department of Neurology, The Second Xiangya Hospital, Central South University, Changsha, 410011 Hunan, China \\ ${ }^{2}$ Department of Orthopaedics, Xiangya Hospital, Central South University, Changsha, 410008 Hunan, China \\ ${ }^{3}$ National Clinical Research Center of Geriatric Disorders, Xiangya Hospital, Central South University, Changsha, \\ 410008 Hunan, China
}

Correspondence should be addressed to Shu-guang Gao; 251469675@qq.com

Received 7 October 2020; Accepted 11 May 2021; Published 17 May 2021

Academic Editor: Laura Baroncelli

Copyright (C) 2021 Miao He et al. This is an open access article distributed under the Creative Commons Attribution License, which permits unrestricted use, distribution, and reproduction in any medium, provided the original work is properly cited.

\begin{abstract}
Neurodegenerative disorders (NDs) are characterized by a gradual loss of neurons and functions that eventually leads to progressive neurological impairment. In view of the heavy burden on the healthcare system, efficient and reliable biomarkers for early diagnosis and therapeutic treatments to reverse the progression of NDs are in urgent need. There has been an increasing interest in using exosomal miRNAs as biomarkers or targeted therapies for neurological diseases recently. In this review, we overviewed the updated studies on exosomal miRNAs as biomarkers and potential therapeutic approaches in NDs, as well as their association with the pathophysiology of this group of disorders, especially Alzheimer's disease (AD), Parkinson's disease (PD), amyotrophic lateral sclerosis (ALS), and Huntington's disease (HD). The exosomal miRNAs that are commonly dysregulated across different NDs or are commonly used as therapeutic candidates were also identified and summarized. In summary, the feasibility of exosomal miRNAs as biomarkers and potential targeted therapy for NDs has been verified. However, due to the limitations of existing studies and the discrepancies across different studies, high quality laboratory and clinical investigations are still required.
\end{abstract}

\section{Introduction}

Neurodegenerative disorders (NDs) are a group of diseases that are characterized by a progressive loss of neurons and functions, including Alzheimer's disease (AD), Parkinson's disease (PD), Huntington's disease (HD), and amyotrophic lateral sclerosis (ALS), among others. Due to gradually increased life expectancy, the prevalence of NDs has shown a continuously increasing trend, associated with huge healthcare costs and significant burden on the healthcare system [1]. The current biomarkers of NDs are subjected to deficiencies such as inability to detect changes in the early or preclinical stage of disease $[2,3]$. Besides, few or no effective therapeutic treatments are available to reverse or cure this group of diseases to date [4]. Therefore, reliable and easily obtainable biomarkers, as well as effective therapeutic approaches, are urgently needed for NDs.

Extracellular vesicles (EVs), which are nanoscale membrane-bound vesicles secreted from cells, consist of exo- somes, microvesicles, and apoptotic bodies based on their intracellular origins $[5,6]$. Since conventional extraction methods of vesicles are usually unable to isolate different classes of EVs, some studies used the terms "EV" and "exosome" interchangeably, but in this review, we primarily focused on exosomes. Exosomes refer to vesicles originate from multivesicular bodies with a diameter of less than $100 \mathrm{~nm}(40-100 \mathrm{~nm})[7,8]$. The cargo content of exosomes is primarily composed of proteins, lipids, DNA, mRNAs, and microRNAs (miRNAs) and plays multiple simultaneous roles throughout the human body [9]. In the central nervous system, exosomes can be secreted into the extracellular space by neurons, neuroglial cells, and neural stem cells to exert a neuroprotective or neurotoxic role and take part in both normal neuronal physiological processes and pathogenic processes [10-14].

As one of the most important types of molecules that are contained in exosomes, miRNAs are a class of small, noncoding RNAs, usually about 22 nucleotides [15]. An individual 


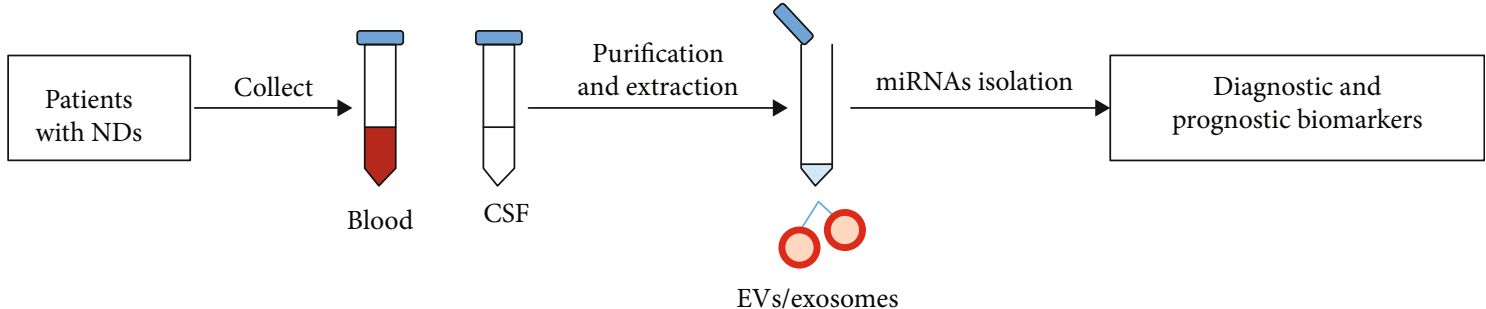

(a)

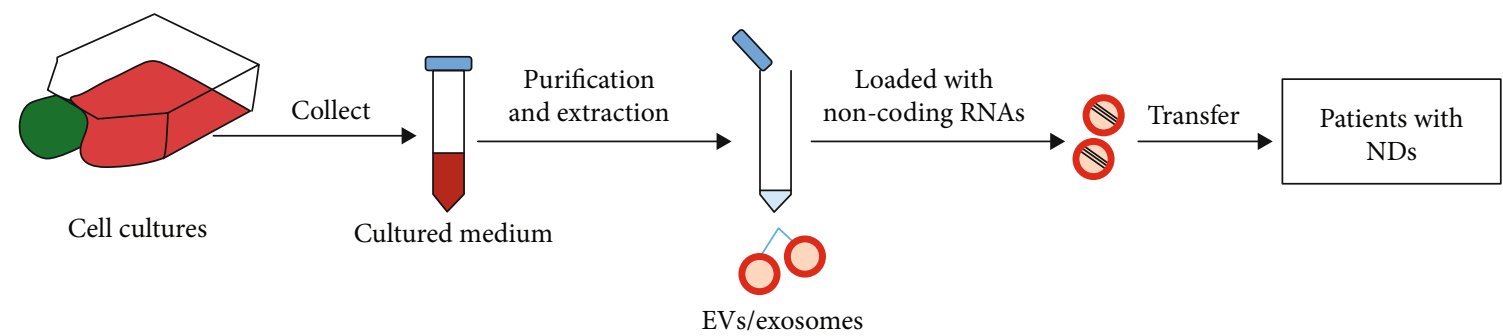

(b)

Figure 1: Collection and clinical applications of EVs/exosomes: (a) EVs/exosomes as therapeutic vehicles; (b) EVs/exosomes as diagnostic and prognostic biomarkers.

miRNA can repress the translation or regulate the degradation of over 100 mRNAs, and one mRNA can be regulated by multiple miRNAs. Thus, miRNAs are involved in most of the key biological processes including cell signaling, neuronal development, maturation, apoptosis, and neural plasticity $[16,17]$.

In this review, we provided an updated overview on the diagnostic and therapeutic potential of exosomal microRNAs for NDs, as well as their association with the pathophysiology of the concerned group of disorders. Exosomal miRNAs that are commonly dysregulated across different NDs or are commonly used as therapeutic candidates were also identified and summarized.

\section{Exosomal miRNA as Biomarkers and Exosomal miRNA-Based Therapy}

Exosomes can be purified through different strategies such as ultracentrifugation, density gradient separation, and immunoaffinity capture methods, but such strategies may slightly affect the exosomal contents including miRNAs [18, 19]. miRNAs are enriched in exosomes compared to cell free serum and plasma [20] and are actively packed into exosomes. According to existing studies, miRNAs may be selectively sorted into exosomes through several distinct mechanisms [6], such as (1) the miRNA motif and heterogeneous nuclear ribonucleoproteins- (hnRNPs-) dependent pathway [21], (2) the neural sphingomyelinase 2(nSMase2-) dependent pathway [22], and (3) the $3^{\prime}$-end of the miRNA sequence-dependent pathway [23]. Encapsulation of miRNAs into EVs can protect those miRNAs from degradation or dilution in the extracellular environment. Therefore, exosomal miRNAs are relatively more stable than miRNAs in serum or plasma [24]. After travelling, these EVs can be efficiently taken up by the targeted cells with specific surface ligands. In this way, exosomes can deliver specific miRNA to the target cells and mediate miRNA exchange between cells, thereby playing a role in cell communication and cell signaling [25]. After being transferred into the recipient cells, miRNAs can then modify the gene expression of recipient cells.

miRNAs may be used as useful diagnostic and prognostic biomarkers of diseases (Figure 1). The expression levels of exosomal miRNAs vary under different physiological and pathological conditions $[2,6]$. Besides, the expression profile of exosomal miRNAs alters in various NDs [26, 27]. Exosomal miRNAs can also be used to discriminate different subtypes of a disease at a high accuracy. For example, the clinical phenotypes of multiple sclerosis (MS) include relapsing-remitting MS (RRMS), secondary progressive MS (SPMS), and primary progressive MS (PPMS) [28]. Ebrahimkhani et al. identified a group of 9 miRNAs that distinguished RRMS from progressive MS. Among the 9 miRNAs, miR15b-5p, miR-374a-5p, miR-30b-5p, miR-342-3p, and miR223-3p were expressed in a higher level in RRMS than in S/PPMS, while miR-433-3p, miR-432-5p, miR-23a-3p, and miR-485-3p were the opposite [28]. In general, exosomal miRNAs have been reported to provide an ideal tool as disease-related biomarkers mainly due to the following advantages: (1) they represent the cell of its origin and alter with the disease; (2) they can be measured easily and reliably with a relatively higher specificity and sensitivity; (3) they are detectable in the early stage of diseases; (4) they can be used to distinguish diseases that exhibit similar clinical symptoms, such as PD and AD, and reduce the risk of misdiagnosis; and (5) they can be used to discriminate different subtypes of a disease [6].

Exosomes can be utilized to deliver drugs or genetic elements (Figure 1) [9]. The delivered miRNA may be able to therapeutically alter gene expressions in certain diseases. For example, Yang et al. reported that the rabies viral 
glycoprotein- (RVG-) exosomes could efficiently deliver miR-124 to the infarct site and promote neurogenesis in ischemic mouse models [29]. The systematically injected exosomal miR-193b-3p could attenuate the neuroinflammatory response in the brains of mice with subarachnoid hemorrhage [30]. Exosomes are deemed promising gene therapy transporters to the brain for the following reasons: (1) exosomes are safe because they do not endogenously replicate [31]; (2) the systematically injected exosomes are able to penetrate the blood brain barrier (BBB) and selectively deliver nucleic acids to the target cells in the brain in a natural way or after modulation [29], and it is feasible to specifically target neural cells by tailoring exosomal membrane proteins [32]; (3) with only biogenic substances, they can travel systemically without stimulating immune responses; and (4) they naturally contain nucleic acids and can protect them from being quickly degraded by ribonuclease in extracellular biological fluids. The miRNA content of exosomes can be modified purposely to enhance their therapeutic effect [29]. Therefore, exosomal miRNAs are attracting increasing attention as potential therapeutic targets for NDs.

2.1. Exosomal miRNAs as Biomarkers and the Potential Therapeutic Approach for AD. The clinical manifestations of AD mainly include deterioration of memory, cognitive decline, and changes in personality and behavior. The pathological changes of $\mathrm{AD}$ are characterized by the accumulation of amyloid plaques and tau containingneurofibrillary tangles in the brain of $\mathrm{AD}$ patients. The formation of amyloid plaques is caused by overproduction of the amyloid $\beta$ peptide $(\mathrm{A} \beta)$. The neurodegenerative changes usually emerge years before the manifestation of clinical symptoms [33].

Dysregulation of miRNAs in the biological body fluid and brain has been found to be associated with the pathological status of $\mathrm{AD}$ [34]. In the review conducted by $\mathrm{Wu}$ et al., it was concluded that 7 miRNAs (miR-29b, miR181c, miR-15b, miR-146a, miR-342-3p, miR-191-5p, and let-7d-5p) were consistently downregulated in more than one $\mathrm{AD}$ study [35]. miR-107 was consistently downregulated in both $\mathrm{AD}$ and mild cognitive impairment (MCI) in two independent cohorts $[36,37]$. miR-132 was found to be consistently upregulated in the plasma/serum in MCI patients when compared to normal controls in 3 different studies [38-40]. In addition, some trials have also reached a similar conclusion. However, the results were inconsistent across most of the studies, possibly due to the differences in miRNA stability and concentration in different samples, the diverse analysis procedures used, and the population differences, which hinders the promotion of peripheral miRNAs as biomarkers for $\mathrm{AD}$ in clinical practice [2].

miRNAs were significantly enriched and more stable in exosomes when compared to cell-free biofluid samples [20]. Alteration of exosomal miRNA as biomarkers has been reported by several studies in $\mathrm{AD}$ patients, suggesting that it could be potentially used to predict $\mathrm{AD}$ status at a high accuracy. From the CSF derived exosome samples, a decrease of miR-16-5p in young-onset $\mathrm{AD}(\mathrm{YOAD},<65 \mathrm{y})$ but not in late-onset $\mathrm{AD}$ (LOAD, $>65 \mathrm{y}$ ), an increase of miR-125b-5p in both YOAD and LOAD, and a decrease of miR-451a and miR-605-5p in both YOAD and LOAD were detected when compared to controls. These miRNAs target the pathways that are relevant to the molecular processes of AD [41]. Significant differences in miR-9-5p and miR-598 detection rates were found between raw and exosome-enriched AD CSF samples but not in controls, implying different exosome trafficking between $\mathrm{AD}$ and control subjects [42]. The expression level of exosomal miR-193b in the CSF and blood samples of $\mathrm{AD}$ patients was reduced when compared to healthy controls but not the total miR-193b level in the blood. Meanwhile, negative correlations were observed between exosomal miR-193b and $\mathrm{A} \beta 42$ in the CSF of AD patients [43]. A total of 20 plasma exosomal miRNAs showed significant differences in the $\mathrm{AD}$ group, among which a panel of 7 miRNAs (miR-185-5p, miR-342-3p, miR-141-3p, miR-342-5p, miR23b-3p, miR-338-3p, and miR-3613-3p) allows highly accurate prediction of the $\mathrm{AD}$ status [44]. Cheng et al. detected 13 serum exosomal miRNAs that were upregulated in $\mathrm{AD}$ (Table 1) and 3 miRNAs downregulated in AD (miR-1306$5 p$, miR-342-3p, and $15 b-3 p$ ) through sequencing analysis [45]. Many of these miRNAs have been shown to be implicated in the AD pathogenesis based on cell and mouse models [45]. For example, hsa-miR-101 targets the $3^{\prime}$ untranslated region of the amyloid precursor protein (APP) to reduce the APP level and the accumulation of $A \beta$ in human cell lines and hippocampal neurons $[46,47]$. The miR-15 family has been observed to regulate tau phosphorylation [48]. miR-424-5p also belongs to the miR-15 family [49]. miR-342-3p is an miRNA that has been proposed as a circulating miRNA biomarker in PD, MS, and CreutzfeldtJakob disease [28]. Aforementioned miRNAs were also found to be correlated with the neuroimaging and neuropsychological examination results. However, since the number of MCI participants was small, none of the exosomal miRNAs was verified as a biomarker for predicting disease progression [45]. The level of serum exosomal miR-223, a neuroinflammation-related miRNA, was correlated with the minimental state examination (MMSE) scores and was found to be significantly decreased in $\mathrm{AD}$ patients. Besides, the level of miR-223 in AD patients at the first clinic visit was significantly lower than that in $\mathrm{AD}$ patients who were currently under medical care, indicating that miR-223 may provide a means to protect nerve cells from apoptosis and can be used to evaluate disease progression [50]. Serum exosomal miR$135 \mathrm{a}$ and miR-384 were detected to be upregulated while miR-193b was downregulated in the serum of AD patients when compared to control subjects. Among the 3 miRNAs, the level of serum exosomal miR-384 was significantly higher in AD patients than in VD (vascular dementia) and PDD (Parkinson's disease with dementia) patients, for which miR-384 appeared to be the best choice among the 3 miRNAs for discriminating $\mathrm{AD}, \mathrm{VD}$, and PDD. However, the combination of the 3 miRNAs outperformed any particular one for the diagnosis of early $\mathrm{AD}$ [51]. Exosomal miR-29c, miR-136-3p, miR-16-2, miR-331-5p, miR-132-5p, and miR-485-5p underwent significant changes in AD CSF when 


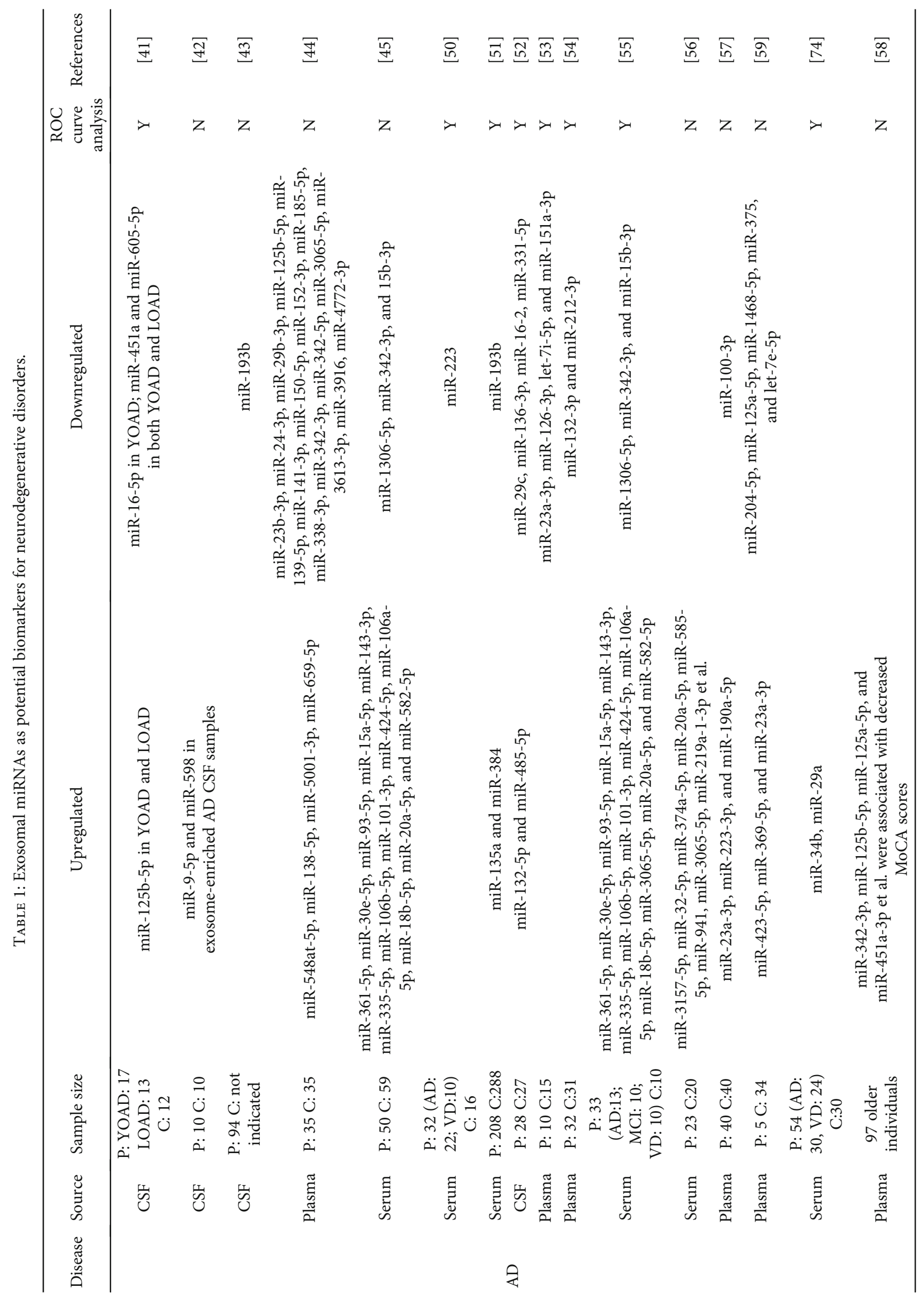




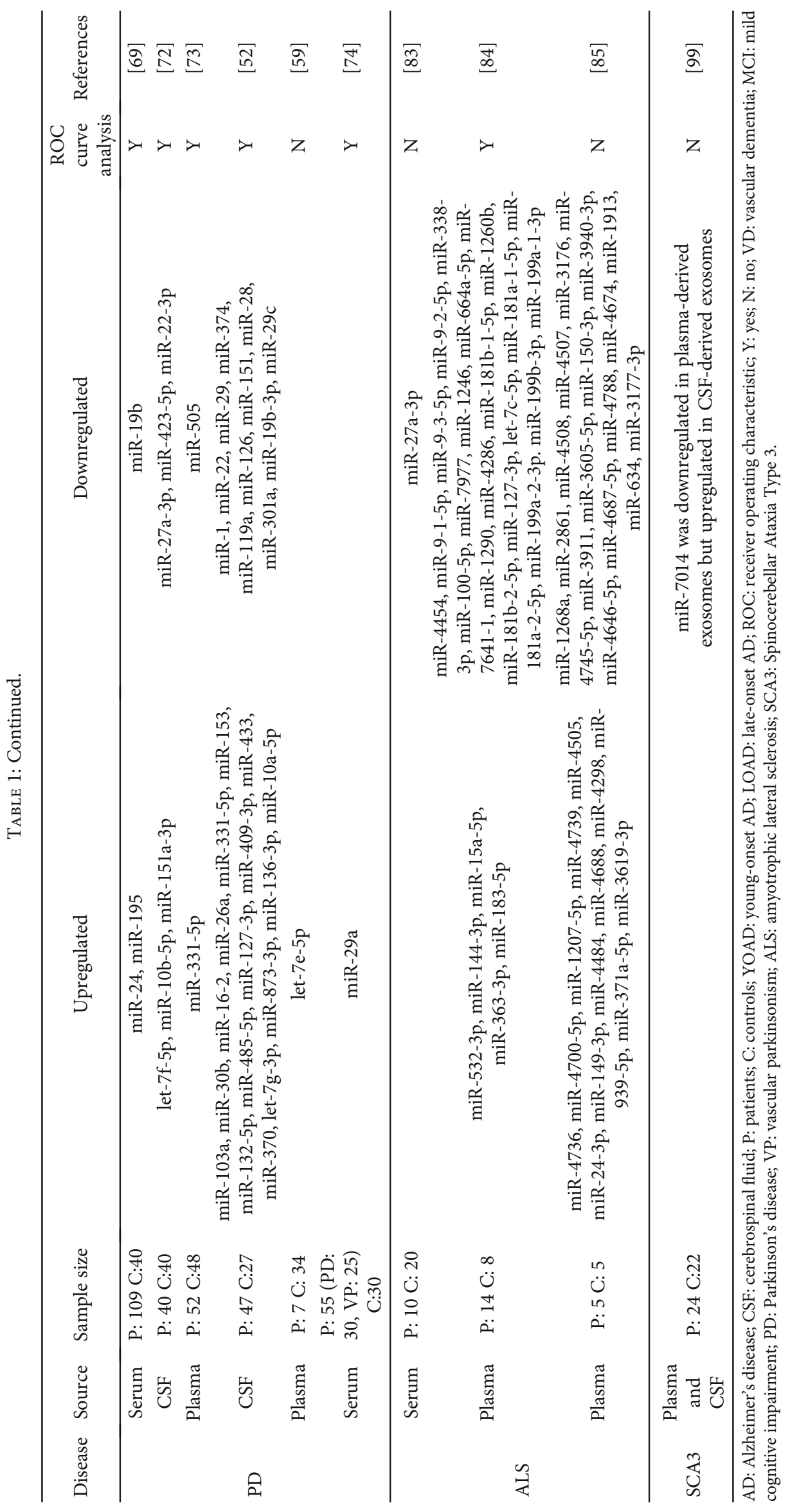


compared to controls [52]. There were 4 miRNAs (miR-23a$3 p$, miR-126-3p, let-7i-5p, and miR-151a-3p) found to be significantly decreased in AD versus controls. The levels of miR451a and miR-21-5p were significantly lower in AD samples than in dementia with Lewy bodies (DLB) samples, for which these two miRNAs can help discriminate AD and DLB. The predicted target gene analysis of these miRNAs was associated with protein phosphorylation, proteasomal pathway, and cell death [53]. miR-132-3p and miR-212-3p had significantly reduced levels in neural-derived plasma exosomes in $\mathrm{AD}$ and showed good sensitivity and specificity to the diagnosis of AD [54]. Li et al. collected serum EV samples from patients with sporadic AD $(n=13)$, MCI $(n=10)$, and VD $(n=10)$, as well as healthy controls $(n=10)$. The expression levels of 4 proteins and 18 miRNAs in EVs were measured by ELISA or qRT-PCR with the results validated in an independent cohort. The expression levels of miR-1306-5p, miR$342-3 p$, and miR-15b-3p were significantly decreased in patients with $\mathrm{AD}$ when compared to controls, but only miR-1306-5p was downregulated in $\mathrm{AD}$ patients (not in VD or MCI patients). A total of 14 miRNAs (Table 1) were significantly upregulated in $\mathrm{AD}$ patients when compared to controls, but only the levels of miR-93-5p, miR-424-5p, and miR-3065-5p were upregulated in $\mathrm{AD}$ patients (not in VD or MCI patients). Even though the sample size was relatively small, the results indicated that the levels of exosomal miR1306-5p, miR-93-5p, miR-424-5p, miR-3065-5p, and protein P-S396-tau might be used to differentiate between controls, $\mathrm{MCI}$, or VD patients and AD patients [55]. Cheng et al. analyzed the association between the miRNAs contained in brain-derived EVs $(n=8)$, the miRNAs from matching total brain homogenate, the miRNAs in peripheral EVs $(n=23)$, and controls $(n=9)$. The results suggested a weak correlation between the miRNAs found dysregulated in the brain and periphery blood of AD subjects when compared to controls. However, those miRNAs that were upregulated in both the brain and serum EVs could be used as liquid biopsy for AD diagnosis (Table 1) [56]. By performing high-throughput sequencing, the miRNA cargo of plasma neural-derived small EVs (NDEVs) from $40 \mathrm{AD}$ patients and 40 controls was tested. Further validation showed that the levels of miR-23a-3p, miR-223-3p, and miR-190a-5p in small NDEVs were found to be significantly upregulated whereas the level of miR-100-3p was significantly downregulated in the AD group when compared to healthy controls [57]. The increased expression levels of 13 plasma exosomal miRNAs were found to be associated with decreased Montreal Cognitive Assessment (MoCA) scores in 97 community dwelling older individuals. Among these 13 cognition-related miRNAs, miR-342-3p, miR-125b-5p, and miR-125a-5p were brain selective, while miR-451a-3p showed the highest expression level [58].

Overall, the exosomal miRNAs that were reported to be differentially expressed in the $\mathrm{AD}$ group in more than one study include miR-361-5p, miR-30e-5p, miR-93-5p, miR15a-5p, miR-143-3p, miR-335-5p, miR-106b-5p, miR-101$3 p$, miR-424-5p, miR-106a-5p, miR-18b-5p, miR-20a-5p, miR-582-5p, miR-1306-5p, miR-342-3p, miR-15b-3p [44, 45, 55], miR-125b-5p [41, 44, 58], miR-139b [43, 51], miR- 3065-5p $[44,55,56]$, miR-23a-3p $[53,57,59]$, and miR$125 \mathrm{a}-5 \mathrm{p}[58,59]$.

RNA interference has emerged as a new promising therapeutic candidate for the treatment of neurodegenerative disorders [60]. The therapeutic potential of RNA drugs delivered by exosomes has aroused a great interest from an increasing number of researchers. Alvarez-Erviti et al. demonstrated the therapeutic potential of exosome-mediated siRNA delivery by transporting exosomal siRNA that targets $B A C E 1$ ( $\beta$-site amyloid precursor protein cleaving enzyme 1 ) to the mouse brain. BACE1 is a protease responsible for the $\mathrm{N}$-terminal cleavage of APP and is a well-known therapeutic target in AD. The researchers first modified dendritic cells to express the lysosome-associated membrane protein 2 (LAMP2) and then fused with RVG to target the central nervous system. Furthermore, they loaded the siRNAs of interest to the derived exosomes. After systematic injection, the siRNA was specifically delivered to the neurons, microglia, and oligodendrocytes mediated by RVG targeted exosomes, resulting in the knockdown of BACE1 and a significant decrease in the total $\beta$-amyloid 1-42 levels [32]. Sarkar et al. demonstrated the overexpression of miR-34a in specific brain regions of $\mathrm{AD}$ patients. The increased level of miR$34 \mathrm{a}$ in the temporal cortex was found to be correlated with the severity of AD pathology. The miR-34a-loaded exosomes secreted by miR-34a-overexpressed neurons deliver miR-34a to recipient neurons. In the target neurons, $\mathrm{miR}-34 \mathrm{a}$ can mediate the concurrent repression of its target genes, which may dedicate to the dysfunction in memory circuits [61]. The expression of miR-29 was found to be associated with DNA damage and cell senescence both in normal and pathological aging and would accumulate during the aging process [62]. The miR-29 family is significantly downregulated in AD and is probably involved in the pathogenesis of the disease. Jahangard et al. transfected the rat bone marrow mesenchymal stem cells (MSC) and HEK-293T cells with vectors carrying the precursor sequences of miR-29. They collected the miR-29 enriched exosomes excreted from the cells after confirming the overexpression of miR-29 and the downregulation of their target genes $B A C E 1$ and $B I M(\mathrm{Bcl}-2$ interacting mediator of cell death (BCL2-like 11)) in the transfected cells. The miR-29 enriched exosomes were then injected into the cornu ammonis area of $\mathrm{A} \beta$-treated model rats. The spatial learning and memory deficits of model rats were prevented after the treatment, indicating that such engineered miR-29 enriched exosomes may have a therapeutic potential. However, animal models cannot mimic all the features of $\mathrm{AD}$, thereby limiting the implication of the findings [63]. Wei et al. reported that the establishment of an AD cell model was accompanied by increased cell apoptosis and decreased miR-223, while the MSC-derived exosomal miR-223 inhibited the neuronal apoptosis of AD cell model by activating the PTEN-PI3K/Akt pathway [64]. Repetitive mild traumatic brain injury (rmTBI) is deemed an important risk factor for AD. The level of microglial exosomal miR-124-3p from injured brain was altered in different phases after rmTBI. After conducting in vitro and in vivo experiments, Ge et al. concluded that microglial exosomal miR-124-3p could alleviate neurodegeneration and cognitive deficits after rmTBI by 
targeting the Rela/ApoE signaling pathway [65]. Even though no overt side effect was reported so far after the execution of exosome-mediated RNA therapy, the long-term impact of the treatment still needs to be further investigated.

\subsection{Exosomal miRNAs as Biomarkers and the Potential} Therapeutic Approach for PD. PD is clinically characterized by resting tremor, rigidity, bradykinesia and postural instability, and various nonmotor symptoms [66]. Exosomes are associated with the spread of $\alpha$-synuclein and inflammatory response and are linked to the progression of $\mathrm{PD}$ pathology in the brain [67]. The dysregulated expression of miRNAs is functionally associated with the pathological process of $\mathrm{PD}$, such as $\alpha$-syn overexpression and spread, Lewy body formation, and neuronal apoptosis [68].

So far, a limited number of studies have been published on exosomal miRNAs as biomarkers for PD. Cao et al. selected 24 candidate human miRNAs that had previously been reported as PD biomarkers. They remeasured the levels of those miRNAs in serum exosomes collected from 109 PD patients and 40 controls and concluded that the expression levels of exosomal miR19b, miR24, and miR195 could support the diagnosis of PD. No correlation was found between miRNAs and the demographics of patients (e.g., age, smoking, drinking, and Hoehn-Yahr scale). By using the Targetscan tool (http://www.targetscan.org), they found several gene targets of the 3 miRNAs to be closely related to the pathological process of $\mathrm{PD}$, including Parkin RBR E3 ubiquitin protein ligase (miR-19b), LRRK2/PARK8 (miR-19b), and ATP13A2/PARK9 (miR-24 and miR-195) [69]. Ren et al. found that the decreased level of miR-195 led to the increase of Rho-associated kinase 1 (ROCK1), which further induced the activation of microglia and triggered neuroinflammation in a cell model of PD, suggesting that miR-195 is a potential therapeutic target for PD [70]. Santos et al. identified 2 CSF exosomal miRNA-based biomarker panels for the early diagnosis of PD. One panel comprised 5 microRNAs (Let-7f-5p, miR-27a-3p, miR-125a-5p, miR-151a-3p, and miR-423-5p), with $90 \%$ sensitivity, $80 \%$ specificity, and $82 \%$ area under the curve (AUC) for the differentiation of the cohorts. miR$27 a-3 p$ was also reported to be decreased in CSF samples of AD patients [71]. Meanwhile, the investigators also identified a panel (miR-10b-5p, miR-22-3p, miR-151a-3p, and $\alpha$-synuclein) with even higher sensitivity, specificity, and AUC. These panels were shown to be associated with the pathways involved in PD pathogenesis such as ubiquitin mediated proteolysis through computational biology analysis [72]. After testing the samples from 52 PD patients and 48 healthy controls, Yao et al. found that the plasma exosomal miR-331-5p was significantly upregulated in PD patients than in controls, while the exosomal miR-505 was downregulated in PD patients. miR-331-5p was mainly packaged in exosomes rather than in the plasma, while miR-505 was mainly expressed in the plasma [73]. A total of 16 exosomal miRNAs were found to be upregulated, and 11 miRNAs were downregulated significantly in PD CSF when compared to normal controls (Table 1). The results from the microRNA assay were validated by TaqMan Real-Time PCR using independent samples [52]. In a recent study conducted by Nie et al., exosomal miRNAs were extracted from the plasma samples collected from $5 \mathrm{AD}$ patients, $7 \mathrm{PD}$ patients, and 34 controls. It was found that 3 miRNAs (miR-423-5p, miR-369-5p, and miR-23a-3p) were significantly elevated, and 5 miRNAs (miR-204-5p, miR-125a-5p, miR-1468-5p, miR-375, and let-7e-5p) were significantly reduced in AD samples when compared to the control group. Only one miRNA, let-7e-5p, was differentially expressed between PD and control. It was found to be elevated in PD samples and reduced in $\mathrm{AD}$ samples, indicating that let-7e-5p can be used as a biomarker to differentiate $\mathrm{AD}$ and $\mathrm{PD}$ [59]. The expression levels of 23 serum miRNAs were investigated in a cohort of 139 patients including $\mathrm{AD}, \mathrm{PD}, \mathrm{VD}, \mathrm{VP}$ (vascular parkinsonism) patients, and healthy controls. The miR-23a showed an increased level in all NDs when compared to controls. The miR-22* and miR-29a were dysregulated in both Alzheimerand Parkinson-like diseases. let-7d, miR-15b, miR-24, miR142-3p, miR-181c, and miR-222 seemed to be associated with Parkinson-like phenotypes, while miR-34b, miR-125b, and miR-130b exhibited altered expressions only in Alzheimer-like disorders [74]. The investigators also compared the expressions of miRNAs in serum exosomes and in serum without exosomes in small groups of patients (5 patients per group). The results showed that miR-23a and miR-125b were only upregulated in the serum deprived of exosomes, while upregulation of miR-34b was observed only in serum exosomes. On the contrary, miR-29a was upregulated in both serum exosomes and serum without exosomes. The results indicated different distributions of miRNAs inside and outside exosomes [74]. Moreover, the exosomal miR-331-5p was reported to be dysregulated in PD by 2 independent studies [52, 73].

Regarding the potential exosomal miRNA therapy, Kojima et al. introduced a set of EXOsomal transfer into cell (EXOtic) devices that enabled efficient and customizable production of designer exosomes in engineered HEK-293T cells. The introduction of EXOtic devices can largely enhance exosome production, specific miRNA packaging, and delivery into the cytosol of target cells. The therapeutic catalase miRNA from engineered exosome-producing cells was able to attenuate neurotoxicity and neuroinflammation in 6hydroxydopamine (6-OHDA) or LPS induced mouse models of PD and could open up new RNA delivery-based therapeutic opportunities for the treatment of PD [75]. To assess the ability of exosomes loaded with $\alpha$-Syn siRNA to decrease $\alpha$ synuclein and its aggregates, Cooper et al. developed modified exosomes expressing RVG and loaded them with $\alpha$-Syn siRNA. Then, they peripherally injected those exosomes into normal mice and transgenic mice expressing the human phosphorylation-mimic S129D $\alpha$-Syn. Significant reduction in brain $\alpha$-Syn mRNA and protein levels, as well as in intraneuronal protein aggregates, was detected in both normal and transgenic mice 7 days after treatment with the $\alpha$-Syn siRNA loaded exosomes [76]. The microRNA-124 loaded nanoparticles were reported to be able to promote the subventricular zone (SVZ) neurogenesis, induce the migration of neurons into the lesioned striatum of the 6-OHDA PD mouse model, and improve the motor performance of 6 OHDA mouse after intracerebral administration [77]. The 
1-methyl-4-phenylpyridinium $\left(\mathrm{MPP}^{+}\right)$is a well-known neurotoxin which can be used to induce cell death in $\mathrm{PD}$ cell models. Shakespear et al. demonstrated that exosomes derived from normal astrocytes, but not from $\mathrm{MPP}^{+}$-stimulated astrocytes, exhibited significant cell-protective effects in $\mathrm{MPP}^{+}$-treated SH-SY5Y cells, as well as in primary mesencephalic dopaminergic and hippocampal neuron cultures. They further confirmed that exosomal miR-200a-3p showed the largest reduction among all the miRNAs expressed in $\mathrm{MPP}^{+}$-stimulated astrocytes through small-RNA sequencing. The astrocyte-derived exosomal miR-200a-3p was found to attenuate apoptotic cell death in $\mathrm{MPP}^{+}$-treated $\mathrm{PD}$ cell models and glutamate-treated hippocampal neuron cultures through downregulation of the mitogen-activated protein kinase kinase 4 (MKK4), which is an important upstream kinase in the c-Jun $\mathrm{N}$-terminal kinase cell death pathway [78]. By upregulating OXR1, the downregulation of exosomal miR-137 could inhibit the oxidative stress injury of neurons, promote neuron viability, and inhibit the apoptosis of neurons in both cell models and mouse models of PD [79].

\subsection{Exosomal miRNAs as Biomarkers and the Potential} Therapeutic Approach for ALS. ALS is a progressive, neurodegenerative disorder with poor prognosis, and its core pathological finding is the loss of upper and lower motor neurons [80]. The clinical manifestation of ALS is heterogeneous, mainly including the upper and lower motor neuron features in limbs and chewing, speaking, and swallowing difficulties $[81,82]$. The pathophysiological processes underlying ALS reflect an interplay between environmental and hereditary factors [81].

Only a few studies have analyzed the expression of exosomal miRNA in ALS. Downregulation of serum exosomal miR-27a-3p was detected in 10 ALS patients when compared to healthy subjects. miR-27a-3p could be transferred by myoblast exosomes to promote osteoblast mineralization and might be involved in ALS development [83]. Analysis of miRNAs in plasma EVs collected from ALS patients revealed elevated levels of 5 miRNAs (especially miR-532-3p, miR144-3p, and miR-15a-5p) and reduced levels of 22 miRNAs (especially miR-4454, miR-9-5p, and miR-338-3p) when compared to controls. Some miRNAs that had previously been reported to be relevant to ALS, including miR-9-5p, miR-183-5p, miR-338-3p, and miR-1246, were found to be deregulated. These ALS-relevant miRNAs were speculated to be associated with the processes such as transcriptional regulation and protein ubiquitination [84]. In plasma neuro-derived EVs collected from ALS patients, 13 miRNAs were detected to be significantly upregulated, and $17 \mathrm{miR}$ NAs were significantly downregulated when compared to healthy controls. Gene ontology analysis revealed that the target genes altered by the dysregulated miRNAs were involved in the synaptic vesicle-related pathway. Most of the miRNAs isolated from neuron-derived EVs were found to be overlapped with the miRNAs expressed in the brain tissue. Particularly, 4 miRNAs in plasma neuro-derived EVs (miR-24-3p, miR-1268a, miR-3911, and miR-4646-5p) were found to be regulated in a similar manner to those in formalin-fixed paraffin-embedded motor cortex samples col- lected from ALS patients. The target genes for the 4 miRNAs partly overlapped in $S T X 1 B, R A B 3 B$, and UNC13A genes. More specifically, UNC13A has been reported to be associated with an increased risk of sporadic ALS [85].

Bonafede et al. reported for the first time that the exosomes from murine adipose-derived stromal cells were able to protect the NSC-34 cells with ALS mutations from oxidative damage and to increase cell viability [86]. The exosomes derived from adipose-derived stem cells have been demonstrated to be able to modulate SOD-1 aggregation and mitochondrial dysfunction in vitro and therefore can be a therapeutic candidate for ALS [87]. Varcianna et al. extracted exosomal miRNAs from human induced astrocytes collected from both ALS patients carrying C9orf72 mutations (C9ORF72-ALS iAstrocytes) and healthy controls. By setting a threshold with $p$ value $\leq 0.05$ and fold change $\geq 1.5$, the investigators identified 64 dysregulated miRNAs (51 upregulated and 13 downregulated) in C9ORF72-ALS iAstrocytes. The dysregulated miRNAs had an impact on neurite network maintenance and motor neuron survival in vitro, suggesting their involvement in the motor neuron death in ALS. In particular, they detected the downregulation of miR-494-3p, which is an upstream target in regulating axonal maintenance and primarily targets Semaphorin 3A (Sema3A). Restoring the level of miR-494-3p can improve motor neuron survival in vitro, which supports miR-494-3p as a potential therapeutic target. However, the difficulty to target specific miRNAs might raise safety concerns for in vivo manipulation [88]. Roy et al. concluded that modulation of the inflammatory-associated miR-124 in mutant copperzinc superoxide dismutase 1 NSC-34 motor neurons and their derived exosomes might be a promising therapeutic strategy for halting motor neuron degeneration in ALS [89].

\subsection{Exosomal miRNAs as Biomarkers and the Potential} Therapeutic Approach for HD. HD is a monogenic, dominantly inherited neurodegenerative disorder that is caused by an abnormal expansion of CAG triplet repeats in the gene huntingtin (HTT). The main clinical manifestation of HD includes involuntary choreiform movements, cognitive impairment, and neuropsychiatric symptoms [90]. Certain miRNAs were detected as biomarkers in the presymptomatic HD gene expansion carriers when treatments may be the most consequential. For example, miR-520f-3p, miR-135b3p, miR-4317, miR-3928-5p, miR-8082, and miR-140-5p were significantly increased in CSF in the prodromal HD group [91].

About $50-60 \%$ of the dysregulated miRNAs in HD (including striatum of mouse HD models, frontal cortex of monkey HD models, and HD patient brain models) were found in exosomes [92], such as miRNA-128a that targets HTT and HIP1 [93]. Other HD related miRNAs, such as miR-22, miR-214, miR-150, miR-146a, and miR-125b, are all found in exosomes [94, 95]. Therefore, miRNAs in exosomes are linked to HD [92].

miR-124 is one of the key miRNAs that is repressed in HD. The researchers first generated a miR-124overexpressing HEK-293 cell line, then harvested miR-124enhanced exosomes from the cells and injected the exosomes 
TABLE 2: Exosomal miRNAs dysregulated in more than one neurodegenerative disorder.

\begin{tabular}{lcc}
\hline Disease & Exosomal miRNAs & References \\
\hline AD and PD & let-7e-5p, miR-151a-3p, miR-423-5p, miR-132-5p, miR-485-5p, & {$[52,53,59,72,74]$} \\
AD and ALS & miR-29a, miR-29c, miR-136-3p, miR-16-2, and miR-331-5p & {$[44,84,85]$} \\
PD and ALS & miR-24-3p and miR-338-3p & {$[52,72,83,84]$} \\
\hline
\end{tabular}

AD: Alzheimer's disease; PD: Parkinson's disease; ALS: amyotrophic lateral sclerosis.

into the striatum of $\mathrm{R} 6 / 2$ transgenic $\mathrm{HD}$ mice [96]. As a result, the expression of the key target gene, RE1-Silencing Transcription Factor, was found to be reduced. However, the exosome treatment had no effect on Dcx protein levels and the behavioral performance of the treated mice, probably due to the limited therapeutic effect of miR-124 or the insufficient dose of miRNAs packed in the exosomes [96]. The engineered microRNA targeting human huntingtin (miHTT) that is delivered via the adeno-associated serotype 5 (AAV5) virus (AAV5-miHTT) has demonstrated a significant huntingtin lowering effect in vitro and in vivo [97]. Sogorb-González et al. developed EVs containing AAV5miHTT from induced pluripotent stem cells- (iPSC-) derived neurons. The therapeutic miHTT molecules within EVs can be taken by other HD neuronal cells in a concentrationdependent manner, and the EVs containing AAV5-miHTT may be therefore used for future gene therapy for HD [98].

2.5. Exosomal miRNAs Dysregulated in Multiple NDs. Exosomal miRNAs that were found to be dysregulated in more than one NDs include let-7e-5p, miR-151a-3p, miR-423-5p, miR-132-5p, miR-485-5p, miR-29a, miR-29c, miR-136-3p, miR-16-2, miR-331-5p in AD and PD [52, 53, 59, 72, 74], miR-24-3p and miR-338-3p in AD and ALS [44, 84, 85], and miR-27a-3p and miR-127-3p in PD and ALS [52, 72, 83, 84]. For Spinocerebellar Ataxia Type 3 (SCA3), miR7014 exhibited a reduced level in plasma-derived exosomes but was upregulated in CSF-derived exosomes and may be a potential biomarker of SCA3 [99] (Table 2).

Exosomal miR-124 has been studied as a candidate therapeutic approach in AD, PD, ALS, and HD models with positive results (Figure 2) $[65,77,89,96]$. miR-124, belonging to the miRNA family, is abundant in the brain and is critical for neuron differentiation and maintenance $[100,101]$. It has also been demonstrated to play a neural protective role in NDs $[102,103]$. Since exosomal miR-124 has been reported to be effective in several ND models, it may provide a novel and promising cell-free therapy for patients with different NDs even though more in vivo and clinical studies are needed.

\section{Conclusions}

In summary, existing studies have verified the feasibility of large-scale clinical applications of exosomal miRNAs as biomarkers. However, there are still discrepancies across different studies, which are in part due to relatively small sample sizes, as well as the technical variability regarding the purification and extraction of exosomal miRNAs. These limitations may be overcome in the future by enlarging the

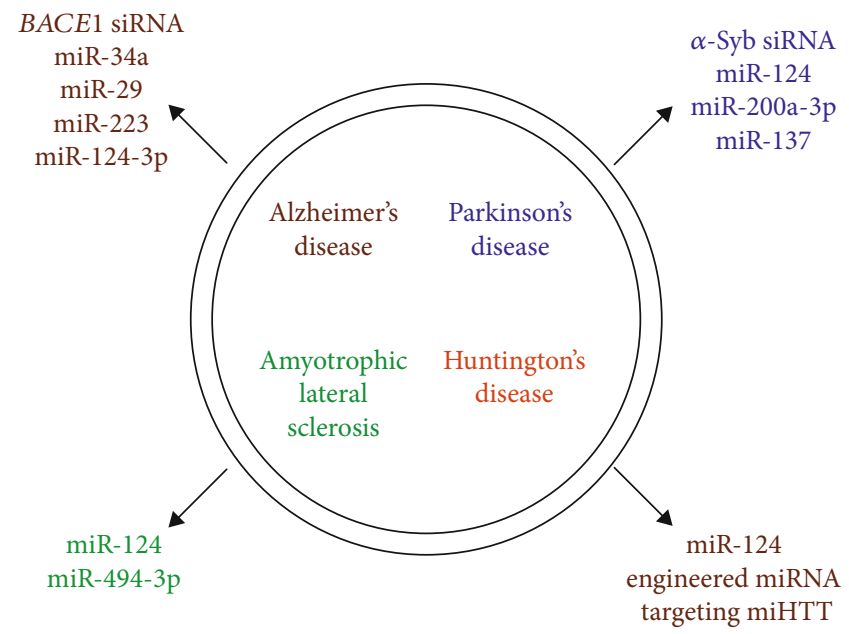

FIgURe 2: Exosomal miRNAs and siRNAs as potential therapeutic strategy for neurodegenerative disorders. miHTT: human huntingtin.

sample size and improving the purification and extraction methods. Exosomal miRNAs are believed to have the potential to become an effective therapeutic strategy, but existing studies are confined to cell and mouse models only. More comprehensive research including high quality laboratory studies with greater insights into the mechanisms of NDs and large-scale clinical studies are still needed for the discovery and further clinical applications of exosomal miRNAs as biomarkers and potential drugs.

\section{Conflicts of Interest}

The authors declare that they have no competing interests.

\section{Authors' Contributions}

Miao He and Shu-guang Gao designed and guided the manuscript. Miao He and Hai-nan Zhang wrote and edited the manuscript. Miao He, Zhen-chu Tang, and Shu-guang Gao reviewed and revised the manuscript. All authors read and approved the final manuscript.

\section{Acknowledgments}

This work was supported by grants from the Youth Program of National Natural Science Foundation of China (81901306, $\mathrm{MH})$, the Huxiang High-Level Talent Gathering Project of Hunan Province (2019RS1011, MH), and the National 
Natural Science Foundation of China (No. 81672225, SGG). We thank the authors of the references for providing the data of the final manuscript.

\section{References}

[1] Y. Hou, X. Dan, M. Babbar et al., "Ageing as a risk factor for neurodegenerative disease," Nature Reviews. Neurology, vol. 15, no. 10, pp. 565-581, 2019.

[2] J. J. Chen, B. Zhao, J. Zhao, and S. Li, "Potential roles of exosomal microRNAs as diagnostic biomarkers and therapeutic application in Alzheimer's disease," Neural Plasticity, vol. 2017, Article ID 7027380, 12 pages, 2017.

[3] L. Wang and L. Zhang, "Circulating exosomal miRNA as diagnostic biomarkers of neurodegenerative diseases," Frontiers in Molecular Neuroscience, vol. 13, p. 53, 2020.

[4] M. D. Paranjpe, A. Taubes, and M. Sirota, "Insights into computational drug repurposing for neurodegenerative disease," Trends in Pharmacological Sciences, vol. 40, no. 8, pp. 565576, 2019.

[5] A. Jarmalaviciute and A. Pivoriunas, "Exosomes as a potential novel therapeutic tools against neurodegenerative diseases," Pharmacological Research, vol. 113, no. Part B, pp. 816-822, 2016.

[6] J. Zhang, S. Li, L. Li et al., "Exosome and exosomal microRNA: trafficking, sorting, and function," Genomics, Proteomics \& Bioinformatics, vol. 13, no. 1, pp. 17-24, 2015.

[7] K. M. Kanninen, N. Bister, J. Koistinaho, and T. Malm, "Exosomes as new diagnostic tools in CNS diseases," Biochimica et Biophysica Acta, vol. 1862, no. 3, pp. 403-410, 2016.

[8] D. Ha, N. Yang, and V. Nadithe, "Exosomes as therapeutic drug carriers and delivery vehicles across biological membranes: current perspectives and future challenges," Acta Pharmaceutica Sinica B, vol. 6, no. 4, pp. 287-296, 2016.

[9] A. Kalani, A. Tyagi, and N. Tyagi, "Exosomes: mediators of neurodegeneration, neuroprotection and therapeutics," Molecular Neurobiology, vol. 49, no. 1, pp. 590-600, 2014.

[10] F. Ciregia, A. Urbani, and G. Palmisano, "Extracellular vesicles in brain tumors and neurodegenerative diseases," Frontiers in Molecular Neuroscience, vol. 10, p. 276, 2017.

[11] F. Antonucci, E. Turola, L. Riganti et al., "Microvesicles released from microglia stimulate synaptic activity via enhanced sphingolipid metabolism," The EMBO Journal, vol. 31, no. 5, pp. 1231-1240, 2012.

[12] D. Frohlich, W. P. Kuo, C. Fruhbeis et al., "Multifaceted effects of oligodendroglial exosomes on neurons: impact on neuronal firing rate, signal transduction and gene regulation," Philosophical Transactions of the Royal Society of London. Series B, Biological Sciences, vol. 369, 2014.

[13] S. Park, E. S. Ahn, and Y. Kim, "Neuroblastoma SH-SY5Y cell-derived exosomes stimulate dendrite-like outgrowths and modify the differentiation of A375 melanoma cells," Cell Biology International, vol. 39, no. 4, pp. 379-387, 2015.

[14] A. D. Pusic, K. M. Pusic, B. L. Clayton, and R. P. Kraig, "IFN $\gamma$-stimulated dendritic cell exosomes as a potential therapeutic for remyelination," Journal of Neuroimmunology, vol. 266, no. 1-2, pp. 12-23, 2014.

[15] D. P. Bartel, "MicroRNAs: genomics, biogenesis, mechanism, and function," Cell, vol. 116, no. 2, pp. 281-297, 2004.
[16] V. N. Kim, J. Han, and M. C. Siomi, "Biogenesis of small RNAs in animals," Nature Reviews. Molecular Cell Biology, vol. 10, no. 2, pp. 126-139, 2009.

[17] V. Ambros, "The functions of animal microRNAs," Nature, vol. 431, no. 7006, pp. 350-355, 2004.

[18] B. J. Tauro, D. W. Greening, R. A. Mathias et al., "Comparison of ultracentrifugation, density gradient separation, and immunoaffinity capture methods for isolating human colon cancer cell line LIM1863-derived exosomes," Methods, vol. 56, no. 2, pp. 293-304, 2012.

[19] K. Rekker, M. Saare, A. M. Roost et al., "Comparison of serum exosome isolation methods for microRNA profiling," Clinical Biochemistry, vol. 47, no. 1-2, pp. 135-138, 2014.

[20] L. Cheng, R. A. Sharples, B. J. Scicluna, and A. F. Hill, "Exosomes provide a protective and enriched source of miRNA for biomarker profiling compared to intracellular and cellfree blood," Journal of Extracellular Vesicles, vol. 3, no. 1, 2014.

[21] C. Villarroya-Beltri, C. Gutierrez-Vazquez, F. Sanchez-Cabo et al., "Sumoylated hnRNPA2B1 controls the sorting of miRNAs into exosomes through binding to specific motifs," Nature Communications, vol. 4, no. 1, p. 2980, 2013.

[22] N. Kosaka, H. Iguchi, K. Hagiwara, Y. Yoshioka, F. Takeshita, and T. Ochiya, "Neutral sphingomyelinase 2 (nSMase2)dependent exosomal transfer of angiogenic microRNAs regulate cancer cell metastasis," The Journal of Biological Chemistry, vol. 288, no. 15, pp. 10849-10859, 2013.

[23] D. Koppers-Lalic, M. Hackenberg, I. V. Bijnsdorp et al., "Nontemplated nucleotide additions distinguish the small RNA composition in cells from exosomes," Cell Reports, vol. 8, no. 6, pp. 1649-1658, 2014.

[24] G. Hu, K. M. Drescher, and X. M. Chen, "Exosomal miRNAs: biological properties and therapeutic potential," Frontiers in Genetics, vol. 3, 2012.

[25] H. Valadi, K. Ekstrom, A. Bossios, M. Sjostrand, J. J. Lee, and J. O. Lotvall, "Exosome-mediated transfer of mRNAs and microRNAs is a novel mechanism of genetic exchange between cells," Nature Cell Biology, vol. 9, no. 6, pp. 654659, 2007.

[26] V. Paschon, S. H. Takada, J. M. Ikebara et al., "Interplay between exosomes, microRNAs and toll-like receptors in brain disorders," Molecular Neurobiology, vol. 53, no. 3, pp. 2016-2028, 2016.

[27] K. S. Sheinerman, J. B. Toledo, V. G. Tsivinsky et al., "Circulating brain-enriched microRNAs as novel biomarkers for detection and differentiation of neurodegenerative diseases," Alzheimer's Research \& Therapy, vol. 9, no. 1, p. 89, 2017.

[28] S. Ebrahimkhani, F. Vafaee, P. E. Young et al., "Exosomal microRNA signatures in multiple sclerosis reflect disease status," Scientific Reports, vol. 7, no. 1, p. 14293, 2017.

[29] J. Yang, X. Zhang, X. Chen, L. Wang, and G. Yang, "Exosome mediated delivery of miR-124 promotes neurogenesis after ischemia," Mol Ther Nucleic Acids, vol. 7, pp. 278-287, 2017.

[30] N. Lai, D. Wu, T. Liang et al., "Systemic exosomal miR-193b$3 p$ delivery attenuates neuroinflammation in early brain injury after subarachnoid hemorrhage in mice," Journal of Neuroinflammation, vol. 17, no. 1, p. 74, 2020.

[31] D. G. Phinney and M. F. Pittenger, "Concise review: MSCderived exosomes for cell-free therapy," Stem Cells, vol. 35, no. 4, pp. 851-858, 2017. 
[32] L. Alvarez-Erviti, Y. Seow, H. Yin, C. Betts, S. Lakhal, and M. J. Wood, "Delivery of siRNA to the mouse brain by systemic injection of targeted exosomes," Nature Biotechnology, vol. 29, no. 4, pp. 341-345, 2011.

[33] A. Serrano-Pozo, M. P. Frosch, E. Masliah, and B. T. Hyman, "Neuropathological alterations in Alzheimer disease," Cold Spring Harbor Perspectives in Medicine, vol. 1, no. 1, 2011.

[34] B. Olsson, R. Lautner, U. Andreasson et al., "CSF and blood biomarkers for the diagnosis of Alzheimer's disease: a systematic review and meta-analysis," Lancet Neurology, vol. 15, no. 7, pp. 673-684, 2016.

[35] H. Z. Wu, K. L. Ong, K. Seeher et al., "Circulating microRNAs as biomarkers of Alzheimer's disease: a systematic review," Journal of Alzheimer's Disease, vol. 49, no. 3, pp. 755-766, 2016.

[36] T. Wang, K. Chen, H. Li et al., "The feasibility of utilizing plasma MiRNA107 and BACE1 messenger RNA gene expression for clinical diagnosis of amnestic mild cognitive impairment," The Journal of Clinical Psychiatry, vol. 76, no. 2, pp. 135-141, 2015.

[37] P. Leidinger, C. Backes, S. Deutscher et al., "A blood based 12miRNA signature of Alzheimer disease patients," Genome Biology, vol. 14, no. 7, p. R78, 2013.

[38] B. Xie, H. Zhou, R. Zhang et al., "Serum miR-206 and miR132 as potential circulating biomarkers for mild cognitive impairment," Journal of Alzheimer's Disease, vol. 45, no. 3, pp. 721-731, 2015.

[39] K. S. Sheinerman, V. G. Tsivinsky, F. Crawford, M. J. Mullan, L. Abdullah, and S. R. Umansky, "Plasma microRNA biomarkers for detection of mild cognitive impairment," Aging (Albany NY), vol. 4, no. 9, pp. 590-605, 2012.

[40] K. S. Sheinerman, V. G. Tsivinsky, L. Abdullah, F. Crawford, and S. R. Umansky, "Plasma microRNA biomarkers for detection of mild cognitive impairment: biomarker validation study," Aging (Albany NY), vol. 5, no. 12, pp. 925-938, 2013.

[41] P. M. McKeever, R. Schneider, F. Taghdiri et al., "MicroRNA expression levels are altered in the cerebrospinal fluid of patients with young-onset Alzheimer's disease," Molecular Neurobiology, vol. 55, no. 12, pp. 8826-8841, 2018.

[42] J. Riancho, J. L. Vazquez-Higuera, A. Pozueta et al., "MicroRNA profile in patients with Alzheimer's disease: analysis of miR-9-5p and miR-598 in raw and exosome enriched cerebrospinal fluid samples," Journal of Alzheimer's Disease, vol. 57, no. 2, pp. 483-491, 2017.

[43] C. G. Liu, J. Song, Y. Q. Zhang, and P. C. Wang, "MicroRNA$193 \mathrm{~b}$ is a regulator of amyloid precursor protein in the blood and cerebrospinal fluid derived exosomal microRNA-193b is a biomarker of Alzheimer's disease," Molecular Medicine Reports, vol. 10, no. 5, pp. 2395-2400, 2014.

[44] G. Lugli, A. M. Cohen, D. A. Bennett et al., "Plasma exosomal miRNAs in persons with and without Alzheimer disease: altered expression and prospects for biomarkers," PLoS One, vol. 10, no. 10, p. e0139233, 2015.

[45] L. Cheng, J. D. Doecke, R. A. Sharples et al., "Prognostic serum miRNA biomarkers associated with Alzheimer's disease shows concordance with neuropsychological and neuroimaging assessment," Molecular Psychiatry, vol. 20, no. 10, pp. 1188-1196, 2015.

[46] J. M. Long and D. K. Lahiri, "MicroRNA-101 downregulates Alzheimer's amyloid- $\beta$ precursor protein levels in human cell cultures and is differentially expressed," Biochemical and Bio- physical Research Communications, vol. 404, no. 4, pp. 889895, 2011.

[47] E. Vilardo, C. Barbato, M. Ciotti, C. Cogoni, and F. Ruberti, "MicroRNA-101 regulates amyloid precursor protein expression in hippocampal neurons," The Journal of Biological Chemistry, vol. 285, no. 24, pp. 18344-18351, 2010.

[48] S. S. Hebert, A. S. Papadopoulou, P. Smith et al., "Genetic ablation of Dicer in adult forebrain neurons results in abnormal tau hyperphosphorylation and neurodegeneration," Human Molecular Genetics, vol. 19, no. 20, pp. 3959-3969, 2010.

[49] J. R. Finnerty, W. X. Wang, S. S. Hebert, B. R. Wilfred, G. Mao, and P. T. Nelson, "The miR-15/107 group of microRNA genes: evolutionary biology, cellular functions, and roles in human diseases," Journal of Molecular Biology, vol. 402, no. 3, pp. 491-509, 2010.

[50] H. Wei, Y. Xu, W. Xu et al., "Serum exosomal miR-223 serves as a potential diagnostic and prognostic biomarker for dementia," Neuroscience, vol. 379, pp. 167-176, 2018.

[51] T. T. Yang, C. G. Liu, S. C. Gao, Y. Zhang, and P. C. Wang, "The serum exosome derived microRNA-135a, -193b, and -384 were potential Alzheimer's disease biomarkers," Biomedical and Environmental Sciences, vol. 31, no. 2, pp. 87-96, 2018.

[52] Y. Gui, H. Liu, L. Zhang, W. Lv, and X. Hu, "Altered microRNA profiles in cerebrospinal fluid exosome in Parkinson disease and Alzheimer disease," Oncotarget, vol. 6, no. 35, pp. 37043-37053, 2015.

[53] A. Gamez-Valero, J. Campdelacreu, D. Vilas et al., "Exploratory study on microRNA profiles from plasma-derived extracellular vesicles in Alzheimer's disease and dementia with Lewy bodies," Translational Neurodegeneration, vol. 8, no. 1, p. 31, 2019.

[54] D. J. Cha, D. Mengel, M. Mustapic et al., "miR-212 and miR132 are downregulated in neurally derived plasma exosomes of Alzheimer's patients," Frontiers in Neuroscience, vol. 13, p. 1208, 2019.

[55] F. Li, X. Y. Xie, X. F. Sui, P. Wang, Z. Chen, and J. B. Zhang, "Profile of pathogenic proteins and microRNAs in plasmaderived extracellular vesicles in Alzheimer's disease: a pilot study," Neuroscience, vol. 432, pp. 240-246, 2020.

[56] L. J. V. Lesley Cheng, K. J. Barnham, C. McLean, C. L. Masters, and A. F. Hill, "Small RNA fingerprinting of Alzheimer's disease frontal cortex extracellular vesicles and their comparison with peripheral extracellular vesicles," Journal of Extracellular Vesicles, vol. 9, no. 1, 2020.

[57] M. Serpente, C. Fenoglio, M. D'Anca et al., "MiRNA profiling in plasma neural-derived small extracellular vesicles from patients with Alzheimer's disease," Cells, vol. 9, no. 6, p. $1443,2020$.

[58] A. Rani, A. O'Shea, L. Ianov, R. A. Cohen, A. J. Woods, and T. C. Foster, "miRNA in circulating microvesicles as biomarkers for age-related cognitive decline," Frontiers in Aging Neuroscience, vol. 9, p. 323, 2017.

[59] C. Nie, Y. Sun, H. Zhen et al., "Differential expression of plasma Exo-miRNA in neurodegenerative diseases by nextgeneration sequencing," Frontiers in Neuroscience, vol. 14, p. $438,2020$.

[60] E. Iranifar, B. M. Seresht, F. Momeni et al., "Exosomes and microRNAs: new potential therapeutic candidates in Alzheimer disease therapy," Journal of Cellular Physiology, vol. 234, no. 3, pp. 2296-2305, 2019. 
[61] S. Sarkar, S. Jun, S. Rellick, D. D. Quintana, J. Z. Cavendish, and J. W. Simpkins, "Expression of microRNA-34a in Alzheimer's disease brain targets genes linked to synaptic plasticity, energy metabolism, and resting state network activity," Brain Research, vol. 1646, pp. 139-151, 2016.

[62] A. P. Ugalde, A. J. Ramsay, J. de la Rosa et al., "Aging and chronic DNA damage response activate a regulatory pathway involving miR-29 and p53," The EMBO Journal, vol. 30, no. 11, pp. 2219-2232, 2011.

[63] Y. Jahangard, H. Monfared, A. Moradi, M. Zare, J. MirnajafiZadeh, and S. J. Mowla, "Therapeutic effects of transplanted exosomes containing miR-29b to a rat model of Alzheimer's disease," Frontiers in Neuroscience, vol. 14, 2020.

[64] H. Wei, Y. Xu, Q. Chen, H. Chen, X. Zhu, and Y. Li, "Mesenchymal stem cell-derived exosomal miR-223 regulates neuronal cell apoptosis," Cell Death \& Disease, vol. 11, no. 4, 2020.

[65] X. Ge, M. Guo, T. Hu et al., "Increased microglial exosomal miR-124-3p alleviates neurodegeneration and improves cognitive outcome after rmTBI," Molecular Therapy, vol. 28, no. 2, pp. 503-522, 2020.

[66] A. Abeliovich and A. D. Gitler, "Defects in trafficking bridge Parkinson's disease pathology and genetics," Nature, vol. 539, no. 7628, pp. 207-216, 2016.

[67] L. Yuan and J. Y. Li, "Exosomes in Parkinson's disease: current perspectives and future challenges," ACS Chemical Neuroscience, vol. 10, no. 2, pp. 964-972, 2019.

[68] L. Leggio, S. Vivarelli, F. L'Episcopo et al., "microRNAs in Parkinson's disease: from pathogenesis to novel diagnostic and therapeutic approaches," International Journal of Molecular Sciences, vol. 18, no. 12, p. 2698, 2017.

[69] X. Y. Cao, J. M. Lu, Z. Q. Zhao et al., "MicroRNA biomarkers of Parkinson's disease in serum exosome-like microvesicles," Neuroscience Letters, vol. 644, pp. 94-99, 2017.

[70] Y. Ren, H. Li, W. Xie, N. Wei, and M. Liu, "MicroRNA195 triggers neuroinflammation in Parkinson's disease in a Rhoassociated kinase 1dependent manner," Molecular Medicine Reports, vol. 19, no. 6, pp. 5153-5161, 2019.

[71] C. Sala Frigerio, P. Lau, E. Salta et al., "Reduced expression of hsa-miR-27a-3p in CSF of patients with Alzheimer disease," Neurology, vol. 81, no. 24, pp. 2103-2106, 2013.

[72] M. C. T. Dos Santos, M. A. Barreto-Sanz, B. R. S. Correia et al., "miRNA-based signatures in cerebrospinal fluid as potential diagnostic tools for early stage Parkinson's disease," Oncotarget, vol. 9, no. 25, pp. 17455-17465, 2018.

[73] Y. F. Yao, M. W. Qu, G. C. Li, F. B. Zhang, and H. C. Rui, "Circulating exosomal miRNAs as diagnostic biomarkers in Parkinson's disease," European Review for Medical and Pharmacological Sciences, vol. 22, no. 16, pp. 5278-5283, 2018.

[74] C. Barbagallo, G. Mostile, G. Baglieri et al., "Specific signatures of serum miRNAs as potential biomarkers to discriminate clinically similar neurodegenerative and vascularrelated diseases," Cellular and Molecular Neurobiology, vol. 40, no. 4, pp. 531-546, 2020.

[75] R. Kojima, D. Bojar, G. Rizzi et al., "Designer exosomes produced by implanted cells intracerebrally deliver therapeutic cargo for Parkinson's disease treatment," Nature Communications, vol. 9, no. 1, p. 1305, 2018.

[76] J. M. Cooper, P. B. Wiklander, J. Z. Nordin et al., "Systemic exosomal siRNA delivery reduced alpha-synuclein aggregates in brains of transgenic mice," Movement Disorders, vol. 29, no. 12, pp. 1476-1485, 2014.
[77] C. Saraiva, J. Paiva, T. Santos, L. Ferreira, and L. Bernardino, "MicroRNA-124 loaded nanoparticles enhance brain repair in Parkinson's disease," Journal of Controlled Release, vol. 235, pp. 291-305, 2016.

[78] N. Shakespear, M. Ogura, J. Yamaki, and Y. Homma, “Astrocyte-derived exosomal microRNA miR-200a-3p prevents $\mathrm{MPP}(+)$-induced apoptotic cell death through downregulation of MKK4," Neurochemical Research, vol. 45, no. 5, pp. 1020-1033, 2020.

[79] Y. Jiang, J. Liu, L. Chen et al., "Serum secreted miR-137containing exosomes affects oxidative stress of neurons by regulating OXR1 in Parkinson's disease," Brain Research, vol. 1722, p. 146331, 2019.

[80] A. Al-Chalabi, L. H. van den Berg, and J. Veldink, "Gene discovery in amyotrophic lateral sclerosis: implications for clinical management," Nature Reviews. Neurology, vol. 13, no. 2, pp. 96-104, 2017.

[81] R. H. Brown and A. Al-Chalabi, "Amyotrophic lateral sclerosis," The New England Journal of Medicine, vol. 377, no. 2, pp. 162-172, 2017.

[82] D. R. Beers and S. H. Appel, "Immune dysregulation in amyotrophic lateral sclerosis: mechanisms and emerging therapies," Lancet Neurology, vol. 18, no. 2, pp. 211-220, 2019.

[83] Q. Xu, Y. Zhao, X. Zhou, J. Luan, Y. Cui, and J. Han, "Comparison of the extraction and determination of serum exosome and miRNA in serum and the detection of miR-27a$3 p$ in serum exosome of ALS patients," Intractable Rare Dis Res, vol. 7, no. 1, pp. 13-18, 2018.

[84] D. Saucier, G. Wajnberg, J. Roy et al., "Identification of a circulating miRNA signature in extracellular vesicles collected from amyotrophic lateral sclerosis patients," Brain Research, vol. 1708, pp. 100-108, 2019.

[85] M. Katsu, Y. Hama, J. Utsumi et al., "MicroRNA expression profiles of neuron-derived extracellular vesicles in plasma from patients with amyotrophic lateral sclerosis," Neuroscience Letters, vol. 708, p. 134176, 2019.

[86] R. Bonafede, I. Scambi, D. Peroni et al., "Exosome derived from murine adipose-derived stromal cells: neuroprotective effect on in vitro model of amyotrophic lateral sclerosis," Experimental Cell Research, vol. 340, no. 1, pp. 150-158, 2016.

[87] M. Lee, J. J. Ban, K. Y. Kim et al., “Adipose-derived stem cell exosomes alleviate pathology of amyotrophic lateral sclerosis in vitro," Biochemical and Biophysical Research Communications, vol. 479, no. 3, pp. 434-439, 2016.

[88] A. Varcianna, M. A. Myszczynska, L. M. Castelli et al., "Micro-RNAs secreted through astrocyte-derived extracellular vesicles cause neuronal network degeneration in C9orf72 ALS," eBioMedicine, vol. 40, pp. 626-635, 2019.

[89] J. Roy, D. Saucier, C. O'Connell, and P. J. Morin, "Extracellular vesicles and their diagnostic potential in amyotrophic lateral sclerosis," Clinica Chimica Acta, vol. 497, pp. 27-34, 2019.

[90] P. McColgan and S. J. Tabrizi, "Huntington's disease: a clinical review," European Journal of Neurology, vol. 25, no. 1, pp. 24-34, 2018.

[91] E. R. Reed, J. C. Latourelle, J. H. Bockholt et al., "MicroRNAs in CSF as prodromal biomarkers for Huntington disease in the PREDICT-HD study," Neurology, vol. 90, no. 4, pp. e264-e272, 2018. 
[92] J. K. T. Wang, P. Langfelder, S. Horvath, and M. J. Palazzolo, "Exosomes and homeostatic synaptic plasticity are linked to each other and to Huntington's, Parkinson's, and other neurodegenerative diseases by database-enabled analyses of comprehensively curated datasets," Frontiers in Neuroscience, vol. 11, 2017.

[93] J. Kocerha, Y. Xu, M. S. Prucha, D. Zhao, and A. W. Chan, "microRNA-128a dysregulation in transgenic Huntington's disease monkeys," Molecular Brain, vol. 7, no. 1, p. 46, 2014.

[94] A. Jovicic, J. F. Zaldivar Jolissaint, R. Moser, F. S. S. Mde, and R. Luthi-Carter, "MicroRNA-22 (miR-22) overexpression is neuroprotective via general anti-apoptotic effects and may also target specific Huntington's disease-related mechanisms," PLoS One, vol. 8, no. 1, p. e54222, 2013.

[95] M. Sinha, J. Ghose, and N. P. Bhattarcharyya, "Micro RNA -214,-150,-146a and-125b target Huntingtin gene," RNA Biology, vol. 8, no. 6, pp. 1005-1021, 2011.

[96] S. T. Lee, W. Im, J. J. Ban et al., "Exosome-based delivery of miR-124 in a Huntington's disease model," J Mov Disord, vol. 10, no. 1, pp. 45-52, 2017.

[97] J. Miniarikova, V. Zimmer, R. Martier et al., "AAV5-miHTT gene therapy demonstrates suppression of mutant huntingtin aggregation and neuronal dysfunction in a rat model of Huntington's disease," Gene Therapy, vol. 24, no. 10, pp. 630-639, 2017.

[98] J. M. Marina Sogorb-Gonzalez, A. Valles-sanchez, S. van Deventer, P. Konstantinova, and M. Evers, "Transfer of therapeutic miRNAs within extracellular vesicles secreted from Huntington's disease iPSC-derived neurons (4548)," Neurology, vol. 94, no. 15, 2020.

[99] X. Hou, X. Gong, L. Zhang et al., "Identification of a potential exosomal biomarker in spinocerebellar ataxia type 3/Machado-Joseph disease," Epigenomics, vol. 11, no. 9, pp. 1037-1056, 2019.

[100] J. Y. Yu, K. H. Chung, M. Deo, R. C. Thompson, and D. L. Turner, "MicroRNA miR-124 regulates neurite outgrowth during neuronal differentiation," Experimental Cell Research, vol. 314, no. 14, pp. 2618-2633, 2008.

[101] M. Lagos-Quintana, R. Rauhut, A. Yalcin, J. Meyer, W. Lendeckel, and T. Tuschl, "Identification of tissuespecific microRNAs from mouse," Current Biology, vol. 12, no. 9, pp. 735-739, 2002.

[102] H. Wang, Y. Ye, Z. Zhu et al., "MiR-124 regulates apoptosis and autophagy process in MPTP model of Parkinson's disease by targeting to Bim," Brain Pathology, vol. 26, no. 2, pp. 167-176, 2016.

[103] N. Kanagaraj, H. Beiping, S. T. Dheen, and S. S. Tay, "Downregulation of miR-124 in MPTP-treated mouse model of Parkinson's disease and MPP iodide-treated MN9D cells modulates the expression of the calpain/cdk5 pathway proteins," Neuroscience, vol. 272, pp. 167-179, 2014. 